\title{
A study of the effectiveness of specialist outreach clinics for orthodontic consultation
}

\author{
Are specialist outreach clinics for orthodontic consultation effective? A randomised controlled trial \\ by K. O'Brien, R. Mattick, N. Mandall, J. Wright, F. Conboy and T. Gosden Br Dent J 2001; 191: 203-207
}

\section{Objective}

To develop outreach clinics for orthodontic consultation and evaluate their costs and effectiveness.

\section{Design}

Single centre randomised controlled trial with random allocation of referred patients to outreach or main base consultation appointments.

\section{Setting}

One hospital orthodontic department and three community health centre clinics in Greater Manchester.

\section{Subjects \\ 324 patients who were referred for orthodontic treatment.}

\section{Main outcome measures}

The outcome of consultation, the cost and duration of the visit and the consumer's perceptions of the visit.

\section{Results}

There were no differences in outcome of the consultation. While consumer travel costs and the duration of appointments were significantly higher for the main base clinics, these differences were not great. However, consumers preferred to attend an appointment in an outreach clinic.

\section{Conclusions}

There do not appear to be marked advantages or disadvantages in providing consultation appointments for orthodontics in outreach clinics.

\section{In Brief}

- This study is a randomised controlled trial of the effectiveness of specialist outreach clinics for orthodontic consultation appointment.

- The results are confined to the setting of a large conurbation.

- In this setting there were no marked advantages or disadvantages in terms of cost or outcome of care.

\begin{abstract}
Comment
This study aimed to establish whether 1 outreach clinics for orthodontic consultation provided tangible benefits to either the hospital orthodontic service or referred patients.

The traditional pattems of delivering specialist outpatient services in the National Health Service are increasingly being questioned. This reflects a desire for closer integration between primary and secondary care and attempts to respond to the wishes of patients.

Outreach clinics, in which the hospital based specialist team provides an outpatient diagnostic or treatment service in primary care rather than in a hospital have become increasingly common in the medical specialities. However, the growth of these outreach clinics in general medical practices without systematic information on their cost effectiveness has caused some controversy.
\end{abstract}

The fact that one third of medical paediatric outreach clinics are eventually stopped $^{1}$ reinforces the need for careful scientific evaluation before introducing such schemes.

In this thorough investigation the authors randomly allocated 324 patients to either a hospital orthodontic department or one of three outreach clinics based in community health centres for their initial orthodontic consultation. The outcomes of all the consultations were assessed as well as the costs (to the patient and the NHS) and duration of each visit and the patient's perception of the care they received.

The results show that the only benefits of providing an initial orthodontic consultation service in outreach clinics were a small reduction in the duration of the consultation and travel costs for the patients. When balanced against the hidden costs, which can accrue when a consultant is absent from his or her main base this study does not provide strong evidence to support the use of outreach clinics for initial orthodontic consultation.

It is also interesting to note that irrespective of where they were seen nearly all the patients indicated that they would be prepared to travel to another setting to see a consultant orthodontist. Research of medical outreach clinics has also found that patients are less concerned about the location of their consultation than they are about the interpersonal aspects of the consultation.

\section{Donald J Burden}

Orthodontic Department, Queen's University, Belfast

1. Spencer N J. Consultant paediatric outreach clinics - a practical step in integration. Arch Dis Child 1993; 68: 496-500. 